\title{
Identification of Long Non-Coding RNA-Associated Competing Endogenous RNA Network in the Differentiation of Chicken Preadipocytes
}

\author{
Lan Chen ${ }^{1,2}$, Tao Zhang ${ }^{1,2, *}$, Shanshan Zhang ${ }^{1,2}$, Jinxing Huang ${ }^{1,2}$, Genxi Zhang ${ }^{1,2}$, \\ Kaizhou Xie ${ }^{1,2}$, Jinyu Wang ${ }^{1,2}$, Haiqing $\mathrm{Wu}^{1,2}$ and Guojun Dai ${ }^{1,2}$ \\ 1 College of Animal Science and Technology, Yangzhou University, Yangzhou 225009, China; \\ chenlan9326@163.com (L.C.); zss18352764956@163.com (S.Z.); hjinxing1225@163.com (J.H.); \\ gxzhang@yzu.edu.cn (G.Z.); kzxie@yzu.edu.cn (K.X.); jywang@yzu.edu.cn (J.W.); hqwu@yzu.edu.cn (H.W.); \\ daigj@yzu.edu.cn (G.D.) \\ 2 Joint International Research Laboratory of Agriculture and Agri-Product Safety, Ministry of Education, \\ Yangzhou University, Yangzhou 225009, China \\ * Correspondence: zhangt@yzu.edu.cn; Tel.: +86-0514-87979075
}

Received: 28 August 2019; Accepted: 8 October 2019; Published: 12 October 2019

\begin{abstract}
Emerging evidence indicates that long noncoding RNAs (lncRNAs) play important roles in the regulation of cell differentiation by acting as competing endogenous RNA (ceRNA). However, the regulatory mechanisms of lncRNA and the lncRNA-associated ceRNA network involved in adipogenic differentiation of chicken preadipocytes remain elusive. Here, we first constructed the chicken preadipocyte in vitro induction model. Then, we identified differentially expressed lncRNAs (DELs), miRNAs (DEMis), and mRNAs (DEMs) between differentiated and undifferentiated preadipocytes. Furthermore, we constructed the IncRNA associated ceRNA network by gene expression correlation analysis and target prediction of DELs, DEMis, and DEMs. Finally, we determined twelve candidate lncRNA-miRNA-mRNA interactions from the IncRNA associated ceRNA network. Eight out of the twelve interactions were validated by RT-qPCR, indicating their potential role in the regulation of chicken preadipocytes differentiation. Among the eight interactions, TCONS_00026544-gga-miR-128-1-5p-RASD1, TCONS_00055280-gga-miR-135a-5p-JAM3, TCONS_00055280-gga-miR-135a-5p-GPR133, TCONS_ 00055280-gga-miR-135a-5p-CLDN1, and TCONS_00055280-gga-miR-135a-5p-TMEM123 may promote adipogenic differentiation of chicken preadipocytes while TCONS_00057272-gga-miR-146a-3p-FOXO6, TCONS_00057242-gga-miR-6615-3p-FOXO6, and TCONS_00057242-gga-miR-6615-3p-ENSGALT00000 043224 have the opposite effects. Our results not only provide novel insights into ceRNA roles of lncRNAs in chicken preadipocytes differentiation and but also contribute to a better understanding of chicken fat deposition.
\end{abstract}

Keywords: long noncoding RNA; preadipocytes differentiation; competing endogenous RNA network; chicken

\section{Introduction}

In the past few decades, the growth rate of broilers has been significantly improved due to continuous selection [1]. However, the high growth rate is accompanied by excessive fat deposition [2]. Excessive fat deposition (mainly abdominal fat) could influence the laying rate, carcass yield, feed conversion ratio, hatching rate, and fertility rate. Therefore, more attention is paid to reducing abdominal fat, which has become a major breeding goal in broilers [3]. In addition, study showed that the adipose tissue of chicken has a relative resistance to insulin, and chicken could be used as 
an animal model to study human type 2 diabetes [4]. Thus, understanding the biological basis of the development of chicken adipose tissue can benefit the development of poultry industry and also provide new idea for human biomedical research. In mammals, the molecular and cellular mechanisms of adipose tissue development have been well studied. However, the mechanisms underlying chicken fat tissue development are complex and remain elusive.

Long non-coding RNAs (lncRNAs) are a class of non-coding RNA greater than 200 nucleotides in length. MicroRNAs (miRNAs) are a class of non-coding single-stranded RNA of approximately 22 nucleotides in length encoded by endogenous genes. miRNAs play essential roles in various life activities, including metabolism [5], development, and disease [6]. Chicken abdominal fat tissue development is a result of the increase in cell number (hyperplasia) and the increase in cell size (hypertrophy or preadipocytes differentiation). Recent studies have shown that LncRNAs and miRNAs participate in regulating preadipocytes differentiation in mammals $[7,8]$. Their roles in the differentiation of chicken preadipocytes are still poorly understood.

The competitive endogenous RNA (ceRNA) hypothesis proposes that transcripts can regulate each other at the post-transcription level by competing for shared miRNAs [9]. IncRNA can act as a miRNA sponge via competing endogenous RNA (ceRNA) activity [10], thereby regulating the gene expression of miRNA [11]. Understanding the RNA interaction will lead to significant insights into gene regulatory network in the differentiation of chicken preadipocytes. The role of lncRNA as ceRNA in adipocytes differentiation of mammal is gradually becoming clear. Liu et al. [12] found that lncRNA Gm15290 sponges miR-27b to promote PPAR $\gamma$-induced fat deposition in mice. Li et al. [13,14] confirmed that lncRNA GAS5 negatively regulates the adipogenic differentiation of MSCs and 3T3-L1 cells by modulating miR-18a and miR-21a-5p as ceRNAs. Li et al. [15] reported that lncRNA ADNCR suppresses adipogenic differentiation by targeting miR-204 in bovine. However, the regulatory mechanisms of IncRNA as ceRNA and the lncRNA-associated ceRNA network involved in adipogenic differentiation of chicken preadipocytes remain elusive.

To identify lncRNAs and lncRNA-associated ceRNA network involved in differentiation of chicken preadipocytes, we systematically analyzed the differentially expressed lncRNAs, miRNAs, and mRNAs using RNA-seq and miRNA-seq. We then constructed the IncRNA-associated ceRNA network following the ceRNA hypothesis. Crucial ceRNA interactions were identified based on the interacted miRNAs function and validated using quantitative reverse transcription polymerase chain reaction (RT-qPCR). Our results not only provide novel insights into ceRNA roles of lncRNAs in chicken preadipocytes differentiation and but also contribute to a better understanding of chicken fat deposition.

\section{Materials and Methods}

\subsection{Ethics Statement}

This experiment was performed in accordance with Chinese guidelines for animal welfare, and the animal protocol was approved by the animal welfare committee of Yangzhou University (permit number SYXK (Su) 2012-0029).

\subsection{Cell Culture and Induction}

Three Haiyang Yellow chickens were anaesthetized with sodium pentobarbital and killed at two weeks old. Primary preadipocytes were isolated from the abdominal fat tissue following methods described by Shang et al. [16]. Complete medium (90\% DMEM/F12 medium,10\% fetal bovine serum, 100 units/mL penicillin, and $100 \mu \mathrm{g} / \mathrm{mL}$ streptomycin) was used to culture chicken preadipocytes in a cell incubator with $5 \% \mathrm{CO}_{2}$ at $37^{\circ} \mathrm{C}$. when the cell reached $90 \%$ confluence, the differentiation medium (supplemented with oleic acid $(400 \mu \mathrm{mol} / \mathrm{L})$ ) was used to induce the differentiation of chicken preadipocytes. Undifferentiated ( 0 days) and differentiated ( 6 days) cells were collected. Each interval was carried out for three biological replicates. The differentiation of chicken preadipocytes was 
determined using RT-qPCR and Oil red O staining. After induction for six days, the cells were fixed in $10 \%$ formalin for $30 \mathrm{~min}$ at room temperature. Then the cells were washed using PBS buffer for three times and stained using Oil red $\mathrm{O}$ working solution for eight minutes. Finally, the cells were washed using PBS buffer and observed using a microscope. The expression level of adipocyte marker genes PPARG and FABP4 in undifferentiated and differentiated preadipocytes was determined using RT-qPCR. The HiScript II Q RT SuperMix for qPCR (+gDNA wiper) kit (Vazyme, Nanjing, China) was used to synthesize the first-strand cDNA following the manufacturer's protocol. The AceQ qPCR SYBR Green Master Mix kit (Vazyme, Nanjing, China) was used to perform RT-qPCR following the manufacturer's protocol.

\subsection{RNA Extraction and Library Construction}

Trizol reagent (Tiangen, Beijing, China) was used to extract total RNA from undifferentiated and differentiated chicken preadipocytes (Add $1 \mathrm{~mL}$ of Trizol per $2.5 \times 10^{6}$ cells). The NEBNext Poly(A) mRNA Magnetic Isolation Module kit (New England Biolabs, Ipswich, MA, USA) and NEBNext Small RNA Library Prep Set for Illumina (Multiplex Compatible) kit (New England Biolabs, Ipswich, MA, USA) were used to construct the lncRNA libraries and miRNA libraries following the manufacturer's protocol, respectively. Six lncRNA libraries (three for each group) and six miRNA libraries (three for each group) were constructed. The 12 constructed libraries were sequenced using the Illumina HiSeqTM 4000 sequencing platform (Illumina Inc., San Diego, CA, USA).

\subsection{LncRNA Identification}

The raw data were filtered by removing low-quality reads $(Q$-value $\leq 20)$, reads containing adapters, reads that are all A bases, and reads containing unknown nucleotides ratio greater than $10 \%$. The clean data were aligned with rRNA sequences of chicken using Bowtie2 to remove reads mapped to rRNA [17]. Then the high-quality clean data were mapped to the Gallus gallus reference genome using Tophat2 (version 2.0.3.12) to identify novel transcripts [18] and known lncRNAs. New lncRNAs were predicted based on the novel transcripts using CPC [19], CNCI [20], and SwissProt database [21]. The arguments and the thresholds used to identify lncRNAs were specified in Data S1.

\section{5. miRNA Identification}

We filter the raw data by removing the low-quality reads ( $Q$-value $\leq 20)$, reads containing adapters, reads shorter than $18 \mathrm{nt}$, and reads with polyA. Then reads were aligned with small RNAs in the GenBank [22] and Rfam [23] database using blastall software (version 2.2.25). The clean data was obtained by removing reads that are more than $97 \%$ identical to rRNA, scRNA, snoRNA, snRNA, and tRNA. The bowtie (1.1.2) software was used to identify known miRNAs by aligning clean data with the miRbase database. Furthermore, the MIREAP_v0.2 software (http://sourceforge.net/projects/mireap/) was used to predict novel miRNAs. The arguments and the thresholds used to identify miRNAs were specified in Data S1.

\subsection{Identification of Differentially Expressed Genes}

Fragments per kilobase of transcript per million mapped reads (FPKM) normalization method was used to quantify the expression levels of lncRNAs and mRNAs. Tags per million (TPM) normalization method was used to quantify the expression levels of miRNAs. The edgeR [24] software was used to identify differentially expressed lncRNAs (DELs), mRNAs (DEMs), and miRNAs (DEMis). Genes with $\mathrm{FDR}<0.05$ and $|\log 2 \mathrm{FC}|>1$ were considered as significantly differentially expressed (DEGs).

$$
\mathrm{FPKM}=\frac{\mathrm{C} \times 10^{6}}{\mathrm{NL} / 10^{3}}
$$


Let FPKM be the expression level of transcript $\mathrm{A}$, then $\mathrm{C}$ is the number of sequencing fragments aligned to transcript $\mathrm{A}, \mathrm{N}$ is the total number of sequencing fragments aligned to the reference transcript, and $\mathrm{L}$ is the number of bases of transcript $\mathrm{A}$.

$$
\mathrm{TPM}=\frac{\text { Actual miRNA counts }}{\text { Total counts of clean tags } \times 10^{6}}
$$

\subsection{Construction of LncRNAs-miRNAs-mRNAs ceRNA Regulatory Network}

First, we predicted the DEMis targets within DELs and DEMs using MIREAP_v0.2, miRanda [25], and TargetScan [26] software. Secondly, we calculated the expression correlation between DEMis and its targets (lncRNA-miRNA or miRNA-mRNA) using the Spearman rank correlation coefficient (SCC) [27]. Pairs with SCC smaller than -0.7 were selected as candidate lncRNA-miRNA or miRNA-mRNA pairs. Thirdly, the expression correlation between DELs and DEMs was calculated using SCC, and pairs with SCC greater than 0.9 were selected as candidate ceRNA (IncRNA-mRNA) pairs. At last, the hypergeometric cumulative distribution function test in $\mathrm{R}$ software was used to identify final ceRNA pairs ( $p$-value $<0.05)$. The lncRNA-miRNA-mRNA network was visualized using Cytoscape software v3.6.0 (http://www.cytoscape.org/) based on the target prediction, expression correlation, and hypergeometric cumulative distribution test results. The NetworkAnalyzer plug-in in Cytoscape software was used to calculate the connection degree. Nodes with connection degree greater than average degree of the whole ceRNA network were identified as highly-connected genes. The hypergeometric cumulative distribution function is as follow:

$$
P-\text { value }=1-\sum_{i=0}^{n-1} \frac{\left(\begin{array}{c}
M \\
i
\end{array}\right)\left(\begin{array}{c}
U-M \\
N-i
\end{array}\right)}{\left(\begin{array}{c}
U \\
N
\end{array}\right)}
$$

$\mathrm{n}$ is the number of miRNA that is shared by $\operatorname{lncRNA}$ and mRNA, $\mathrm{U}$ is the number of all miRNAs, $\mathrm{M}$ is the number of target miRNAs of lncRNAs, and $\mathrm{N}$ is the number of target miRNAs of mRNAs.

\subsection{Functional Enrichment Analysis}

To characterize the underlying function of the network, DEMs involved in the lncRNA-associated ceRNA network were performed to Gene Ontology (GO), and Kyoto Encyclopedia of Genes and Genomes (KEGG) enrichment analysis using clusterProfiler package [28] in R software based on the GO (http://www.geneontology.org/) and KO (http://www.kegg.jp/kegg/kegg1.html) databases. Gallus gallus was selected as the reference species for enrichment analysis. GO terms and KEGG pathways with $p$-value $<0.05$ were considered as significantly enriched.

\subsection{Quantitative Reverse Transcription Polymerase Chain Reaction}

In the ceRNA network, three miRNAs were reported to be involved in adipocytes differentiation, including gga-miR-146a, gga-miR-135a, and gga-miR-128-1. gga-miR-6615 and gga-miR-135a were high-connected in the ceRNA network and high-expressed in chicken preadipocytes. These four miRNAs were involved in 12 ceRNA interactions such as TCONS_00026544-gga-miR-128-1-5p-RASD1, TCONS_00057272-gga-miR-146a-3p-FOXO6, TCONS_00057242-gga-miR-6615-3p-FOXO6, TCONS_ 00057242-gga-miR-6615-3p-ENSGALT00000043224, TCONS_00040913-gga-miR-6615-3p-ENSGALT 00000043224, TCONS_00040913-gga-miR-6615-3p-ENSGALT00000050730, TCONS_00038747-gga-miR -6615-3p-ENSGALT00000050730, TCONS_00038748-gga-miR-6615-3p-ENSGALT00000050730, TCONS_ 00055280-gga-miR-135a-5p-JAM3, TCONS_00055280-gga-miR-135a-5p-GPR133, TCONS_00055280gga-miR-135a-5p-CLDN1, TCONS_00055280-gga-miR-135a-5p-TMEM123. RT-qPCR was used to validate these ceRNA interactions. Each reaction was carried out for three biological and technical 
replicates. The primers for RT-qPCR are designed using the online Primer-Blast tool (Table 1). The $2^{-\Delta \Delta \mathrm{Ct}}$ method was used to quantify the expression level of selected genes.

Table 1. Primers for RT-qPCR.

\begin{tabular}{|c|c|}
\hline Gene & Primer Sequences \\
\hline \multirow{2}{*}{ TOCNS_00055280 } & GCAGAACGGATGGTGCCTTTGT \\
\hline & GGCTGTCCTGGCTGAAGATGGA \\
\hline \multirow{2}{*}{ TOCNS_00057242 } & СССТTСТССGСТCACAGTCСТT \\
\hline & CGCTTCGGCTGTAAACGTCCAT \\
\hline \multirow{2}{*}{ TOCNS_00057272 } & GGCTACCCGTCTCCTCCAAGAT \\
\hline & GTGCCTCGTTACGCCTGATTGA \\
\hline \multirow{2}{*}{ TOCNS_00026544 } & ACGGAGATGCTGCGGTTATGC \\
\hline & AACAAACCCAACCCGTTCCCAG \\
\hline \multirow{2}{*}{ ENSGALT00000043224 } & TCCAAGAAGGCGGTCACCAAGA \\
\hline & GCACCTGCTTCAGCACCTTGT \\
\hline \multirow{2}{*}{ FOXO6 } & CAGCAGACCTGGACCTGGACAT \\
\hline & CGCCGAGTCGAAGTTGAAGTCC \\
\hline \multirow{2}{*}{ RASD1 } & CCTCGTGTTCAGCCTGGACAAC \\
\hline & CGGCACCTCGATGTTCTCCTTG \\
\hline \multirow{2}{*}{ GPR133 } & GTGAGCACCATCCGCAACCAA \\
\hline & AAGGCACCGTTCCAGGACTGAA \\
\hline \multirow{2}{*}{ JAM3 } & GGAAGTCCTCCTCGCAGCAGTT \\
\hline & AACAAGCCAGGTGCCCACTCT \\
\hline \multirow{2}{*}{ CLDN1 } & GCTGATTGCTTCCAACCAGGCT \\
\hline & GCACACGGCTCTCCTTGTCTAC \\
\hline \multirow{2}{*}{ TMEM123 } & CGTACCACTCGAGAAGAGGC \\
\hline & CAGCTGTGACAGGATGGGTT \\
\hline gga-miR-6615-3p & $\begin{array}{l}\text { GTCGTATCCAGTGCAGGGTCCGAGGTATTCGCACTGGATACGACTGTGGA } \\
\text { CGCGTGGCACTGATGTGTTC }\end{array}$ \\
\hline \multirow{2}{*}{ gga-miR-146a-3p } & GTCGTATCCAGTGCAGGGTCCGAGGTATTCGCACTGGATACGACCTGAAG \\
\hline & CGACCCATGGGGCTCAGTT \\
\hline gga-miR-128-1-5p & GTCGTATCCAGTGCAGGGTCCGAGGTATTCGCACTGGATACGACTCTCAG \\
\hline \multirow{2}{*}{ gga-miR-135a-5p } & $\begin{array}{l}\text { GCGGGGCCGTAACACTGT } \\
\text { GTCGTATCCAGTGCAGGGTCCGAGGTATTCGCACTGGATACGACTCACAT }\end{array}$ \\
\hline & CGCGTATGGCTTTTTATTCCT \\
\hline
\end{tabular}

\subsection{Statistical Analysis}

The SPSS for Windows software (version 22, SPSS, Inc.) and Excel software (Microsoft Corp.) were used to analyze experimental data. The SPSS was used to test the normal distribution of the RT-qPCR data. The RT-qPCR data conformed to the normal distribution (Data S2). Student t-test was used to analyze the difference in RT-qPCR data. All tests were performed at least in triplicate. The $p<0.05$ value was considered to indicate a statistically significant difference.

\section{Results}

\subsection{Induction of Chicken Preadipocytes}

The cells were induced to differentiation with differentiation medium supplemented with oleic acid. The oil red o staining and RT-qPCR were used to determine cell differentiation. We found that, after six days of induction, preadipocytes were fully differentiated and filled with large lipid droplets (Figure 1A,B). The relative expression level of adipocyte marker genes FABP4 and PPARG significantly increased (Figure 1C). 
A

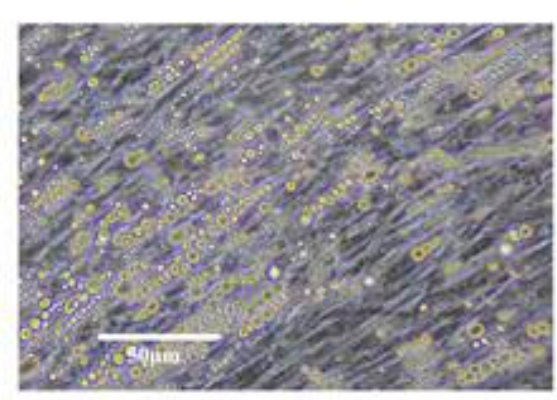

B

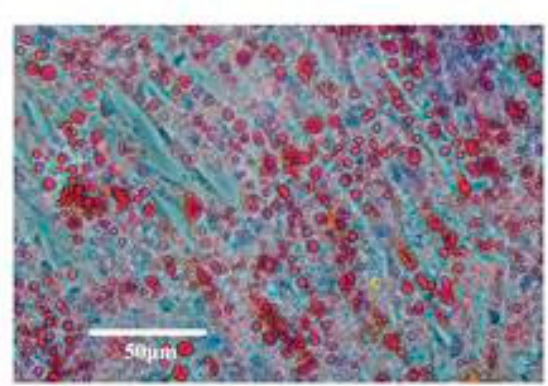

C

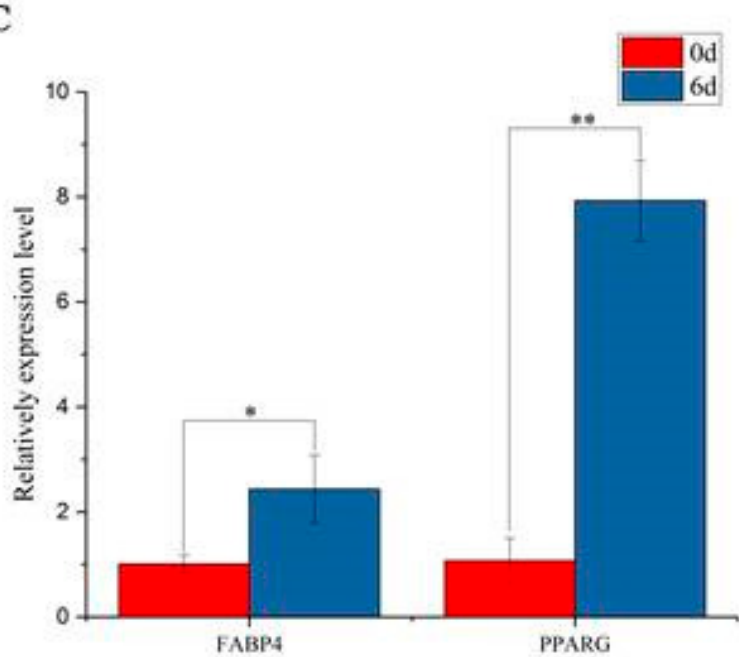

Figure 1. Differentiation of chicken preadipocytes. (A) Differentiated preadipocytes before oil red o staining. (B) Differentiated preadipocytes stained using Oil red O. (C) The relative expression level of adipocyte marker genes before and after differentiation. The relative expression level of FABP4 and PPARG genes significantly increased post-differentiation, indicating that the chicken preadipocytes were successfully induced. *denotes $p<0.05,{ }^{* *}$ denotes $p<0.01$.

\subsection{Identification of Differentially Expressed lncRNAs, miRNAs, and mRNAs}

A total of 3881 lncRNAs were identified in chicken preadipocytes (Data S3). A correlation heat map was used to show the relationship between samples, which indicates that samples in the same group were highly correlated (Figure 2A). Differentially expressed genes between undifferentiated and differentiated preadipocytes were identified using edgeR software. Consequently, 235 DELs, 145 DEMis, and 660 DEMs were identified on the basis of RNA-Seq and small RNA-seq data (Table S1). Heatmaps generated from the expression of DELs, DEMis, and DEMs were used to show the expression patterns of these genes between undifferentiated and differentiated preadipocytes (Figure 2B-D). 
A
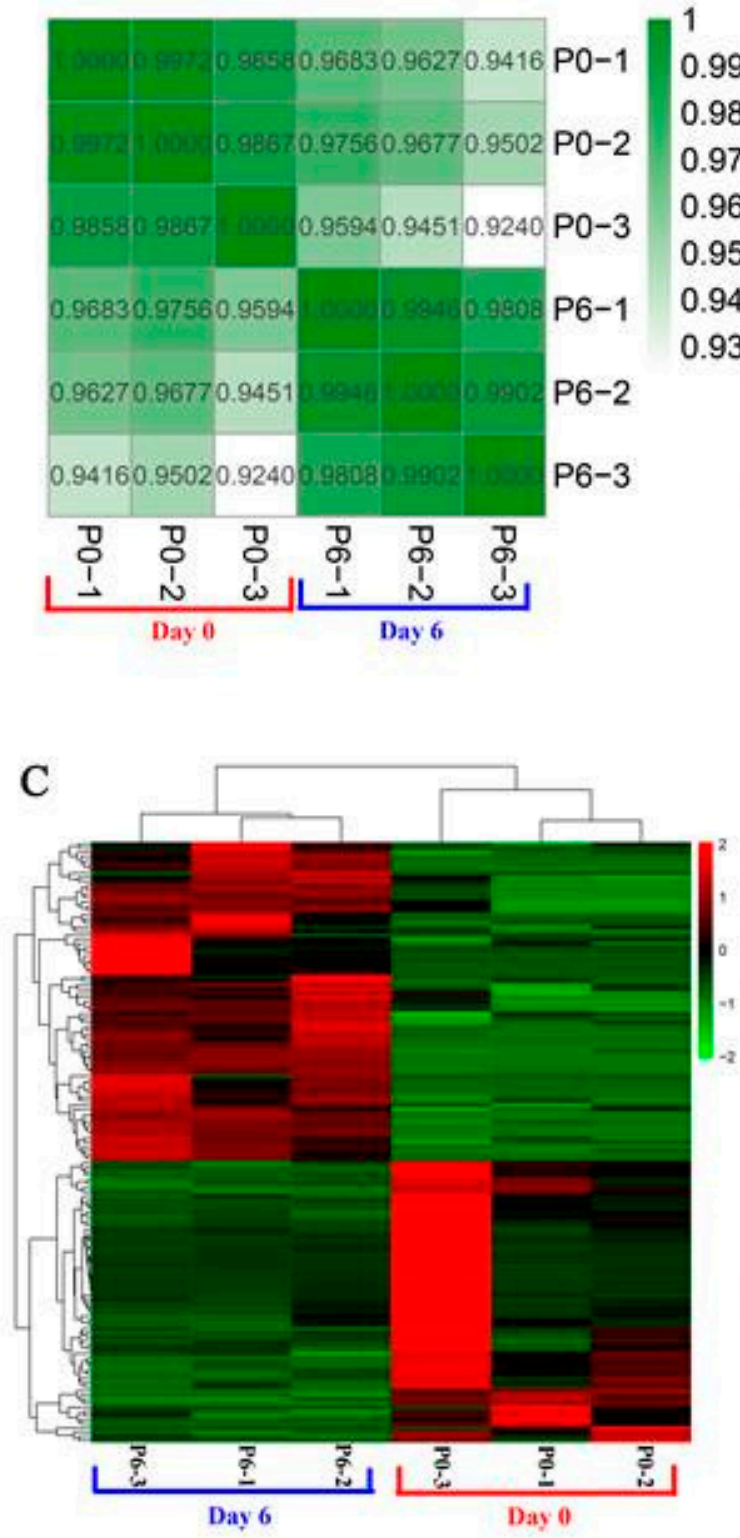

B

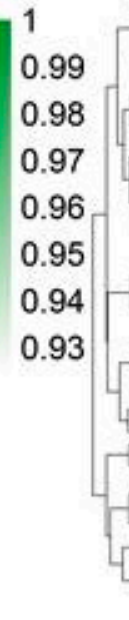

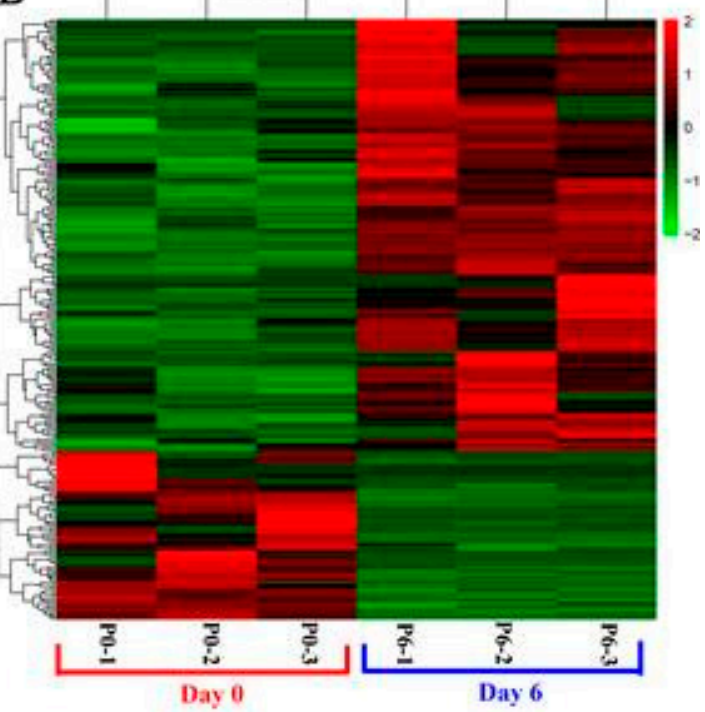

D

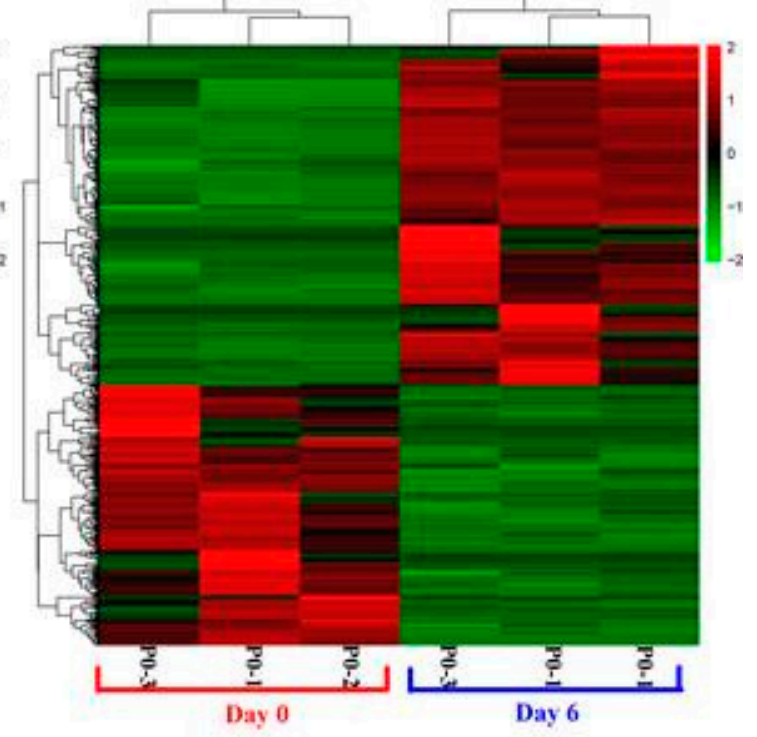

Figure 2. Heatmaps of sample correlation and differentially expressed genes. (A) Sample correlation heatmap. Dark green indicates a high correlation. (B) Heatmap for differentially expressed long noncoding RNAs (lncRNAs). (C) Heatmap for differentially expressed microRNAs (miRNAs). (D) Heatmap for differentially expressed mRNAs. Red indicates highly expressed genes, and green indicates lowly expressed genes.

\subsection{Construction of ceRNA Network Related to Preadipocytes Differentiation}

By the microRNA response elements (MREs) prediction, we obtained 2262 interactions between 145 DEMis and 232 DELs, as well as 999 interactions between 139 DEMis and 192 DEMs. Based on SCC, 438 interactions between DEMis and DELs, 297 interactions between DEMis and DEMs, and 2883 interactions between DELs and DEMs were identified (Table S2). Combined the MREs prediction and SCC results, DELs and DEMs that shared common DEMis were selected as candidate ceRNAs and tested using hypergeometric cumulative distribution function. In total, 251 ceRNAs (lncRNA-miRNA-mRNA) $(p<0.05)$ of 96 lncRNAs, 71 miRNAs, and 84 mRNAs were identified 
(Table S3). The constructed lncRNA-miRNA-mRNA network was visualized using the Cytoscape software (Figure 3). Network analysis showed that the average degree of the whole network was 4.9.

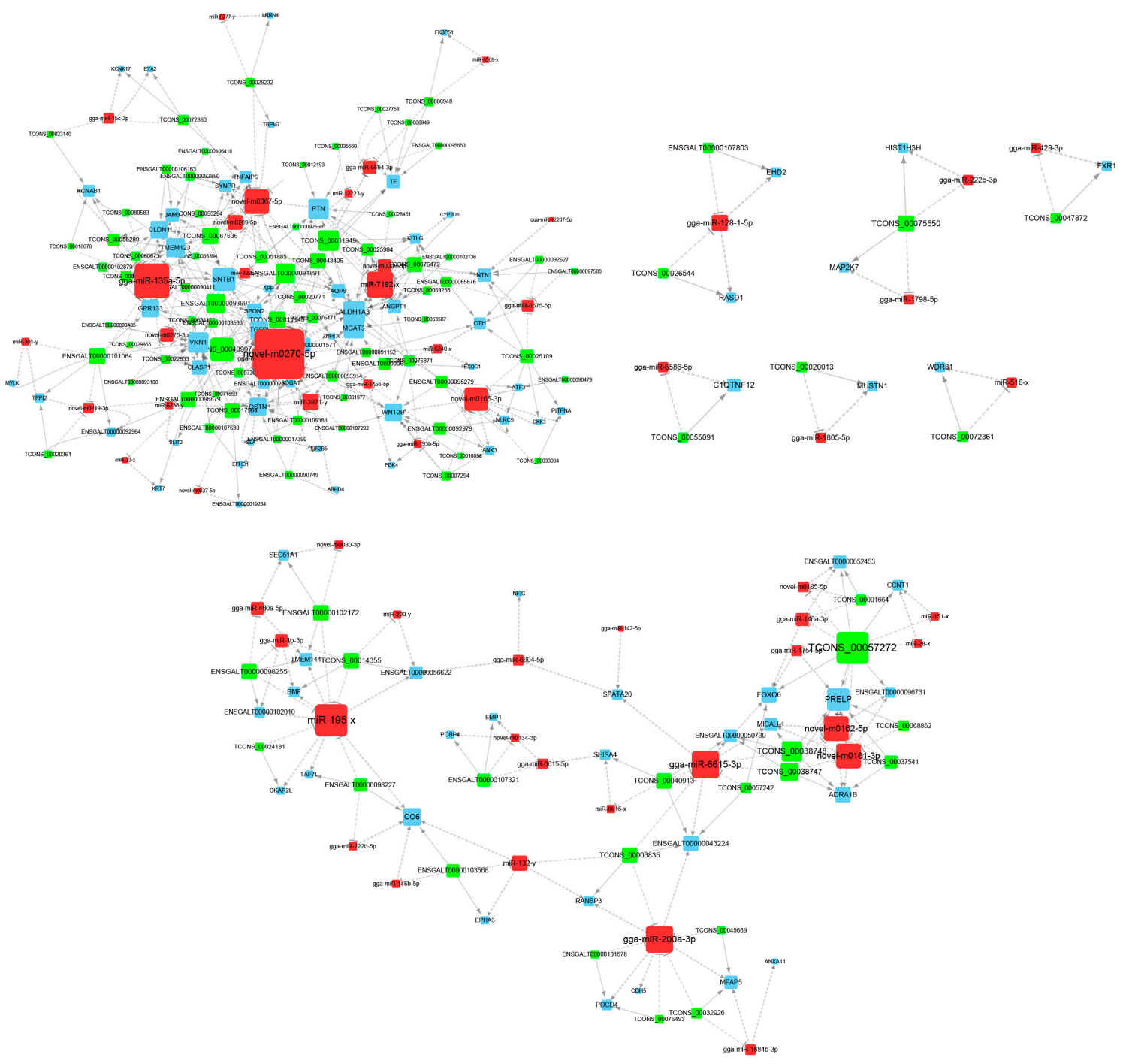

Figure 3. Visualization of the lncRNA-miRNA-mRNA network. Green nodes indicate lncRNAs, red nodes indicate miRNAs, and blue nodes indicate mRNAs, respectively. Node size is proportional to the connection degree. Connection degree shows the number of connected nodes with the individual node. The higher the degree of a node, the more important the node in the network. Hub genes are the nodes with higher degree i.e., nodes with more connections (degree $>4.9$ ).

\subsection{Functional Enrichment Analyses}

To further understand the underlying function of lncRNA-associated ceRNA network, GO and KEGG pathway analyses were performed on mRNAs involved in the ceRNA network. GO analyses showed that genes involved in the ceRNA network are associated with response to external stimulus, positive regulation of cell differentiation, and positive regulation of multicellular organismal biological processes (Figure 4). KEGG pathway analyses identified four significant enriched pathways, including pantothenate and CoA biosynthesis, mineral absorption, steroid hormone biosynthesis, and vascular smooth muscle contraction (Figure 5). 


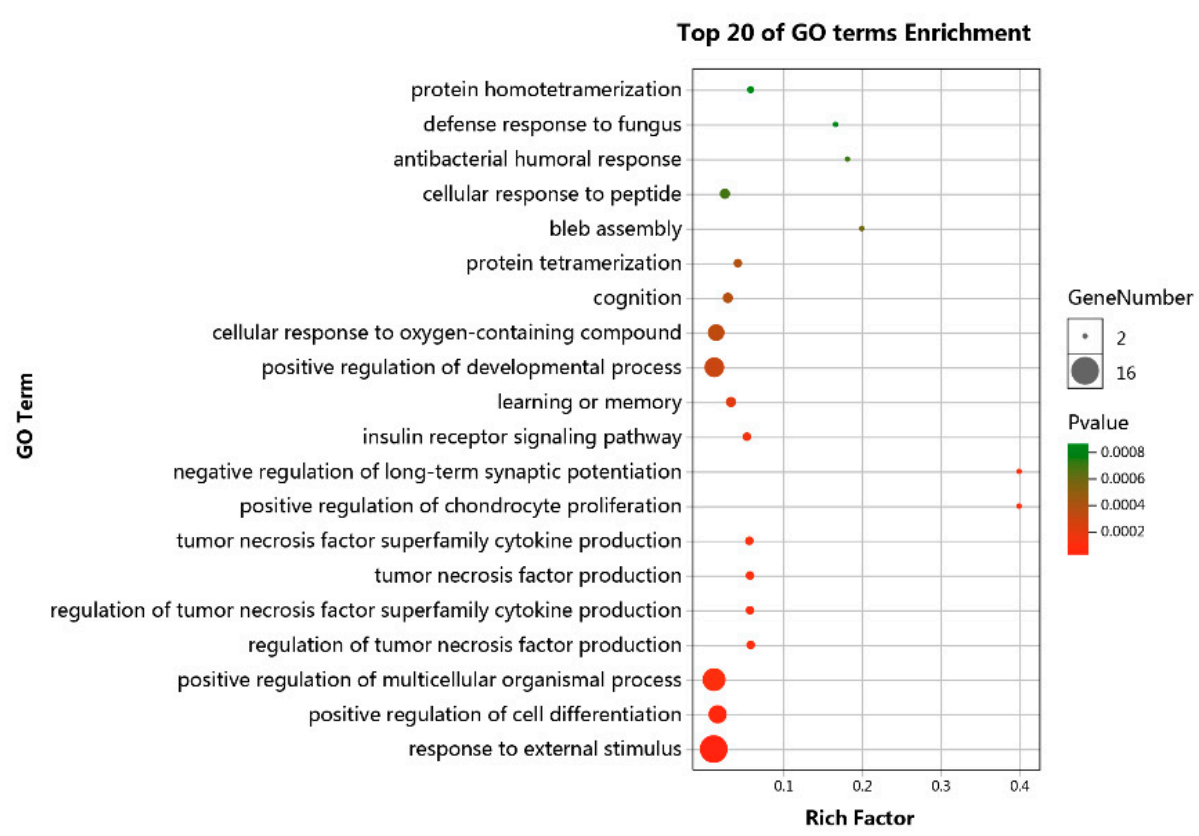

Figure 4. GO analyses of mRNAs involved in the lncRNA-related competing endogenous RNA (ceRNA) network.

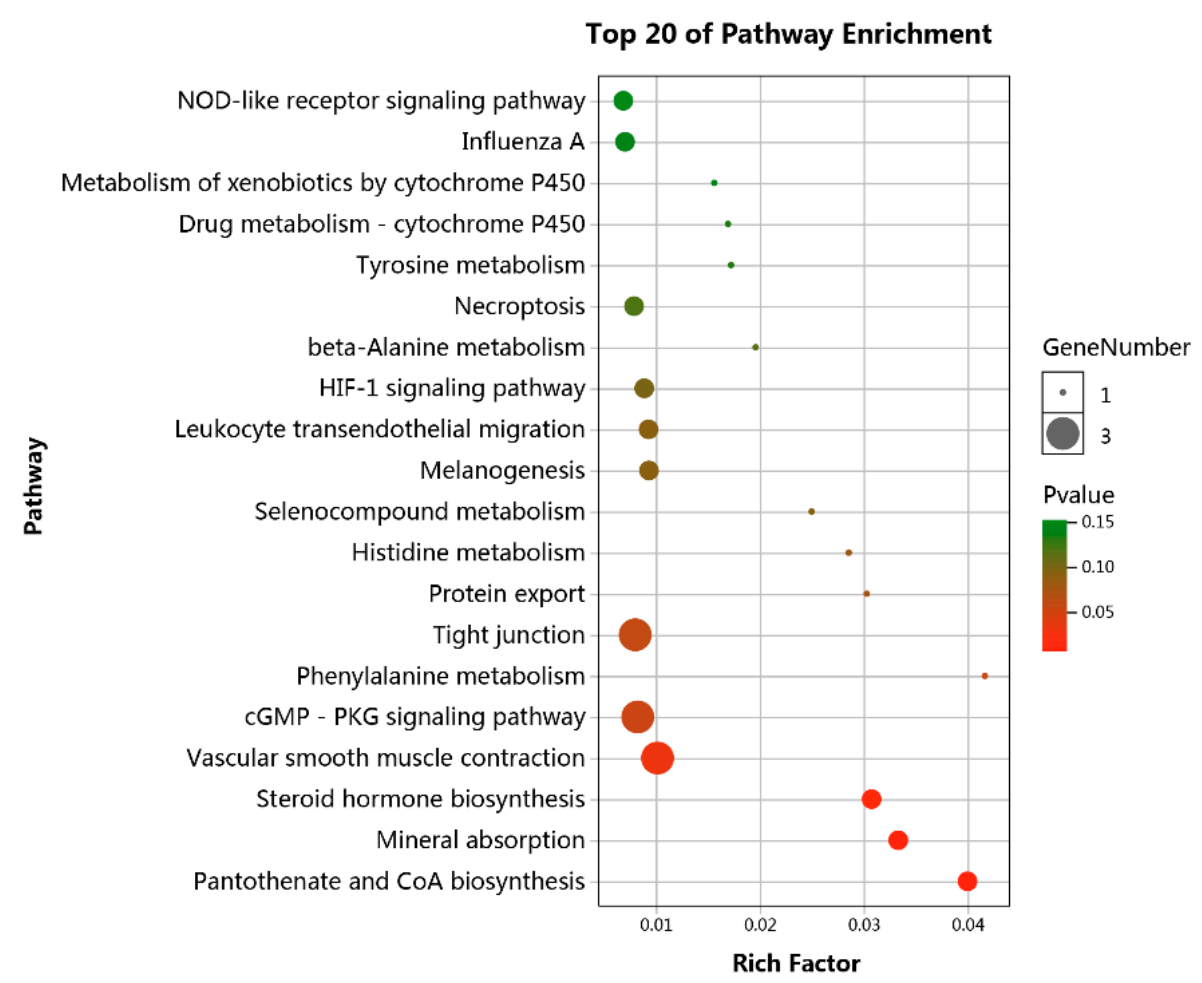

Figure 5. KEGG pathway analyses of mRNAs involved in the lncRNA-related ceRNA network.

\subsection{Identification and RT-qPCR Validation of Crucial Genes}

In our study, miRNAs with TPM greater than 4.5 (more than two-thirds of the DEMis expression is lower than 4.5) were identified as highly expressed. Genes with connection degree greater than 4.9 were identified as highly connected. gga-miR-135a and gga-miR-6615 were high-connected in the network (degree > 4.9) and high-expressed in chicken preadipocytes (TPM > 4.5). gga-miR-146a, gga-miR-135a, and gga-miR-128-1 were reported to participate in the regulation of 3T3-L1 differentiation [29-31], suggesting their potential roles in chicken preadipocytes differentiation. Thus, ceRNA interactions 
containing these four miRNAs were identified as crucial ceRNA interactions. Seven lncRNAs and seven mRNAs interacting with these four miRNAs formed twelve ceRNA interactions (Figure 6, Table 2). Eight out of twelve ceRNA interactions were validated using RT-qPCR, demonstrating high consistency between RT-qPCR and RNA-seq results (Figure 7).
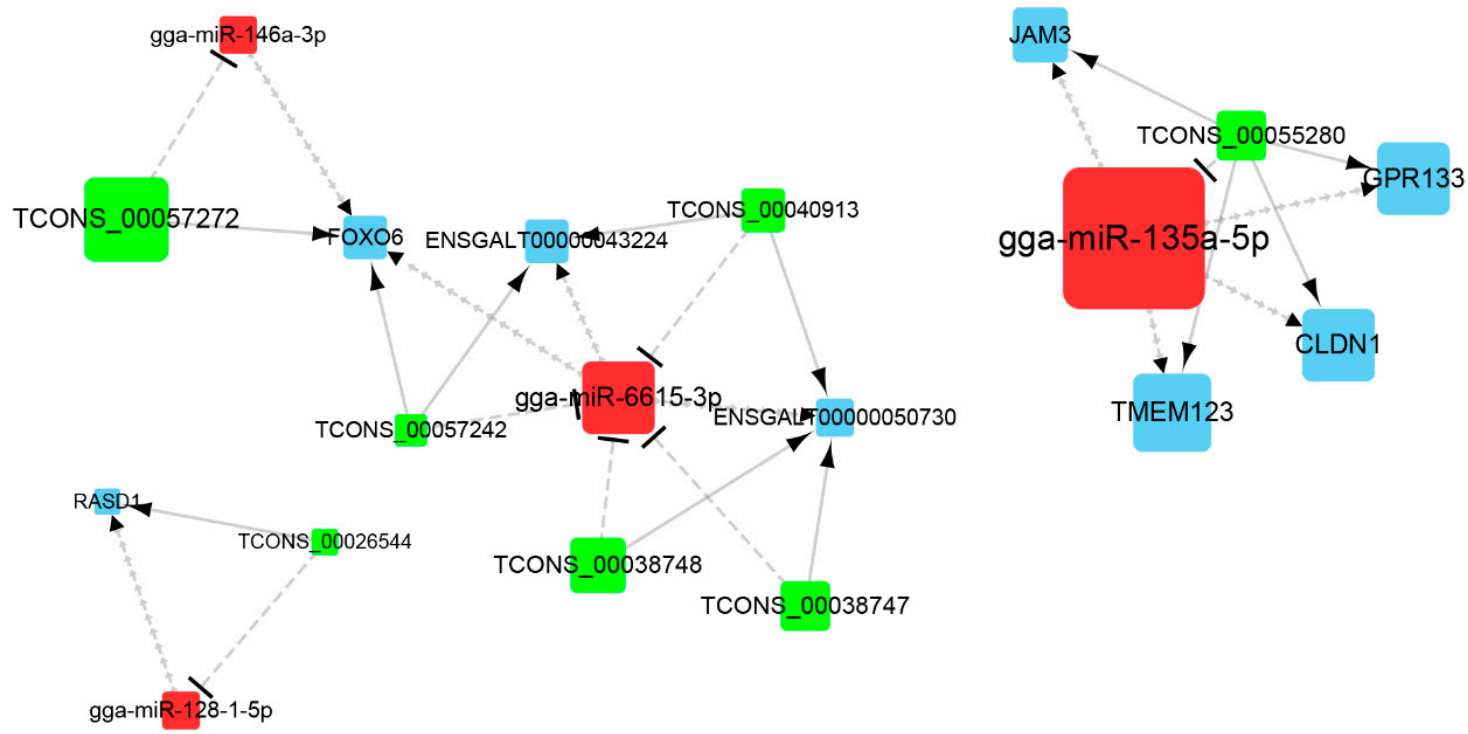

Figure 6. Visualization of crucial genes in the ceRNA network. Green, red, and blue nodes indicate lncRNAs, miRNAs, and mRNAs, respectively.

Table 2. The crucial ceRNA Interactions.

\begin{tabular}{ccc}
\hline IncRNA & miRNA & mRNA \\
\hline TCONS_00026544 & gga-miR-128-1-5p & RASD1 \\
TCONS_00057272 & gga-miR-146a-3p & FOXO6 \\
TCONS_00057242 & gga-miR-6615-3p & FOXO6 \\
TCONS_00057242 & gga-miR-6615-3p & ENSGALT00000043224 \\
TCONS_00040913 & gga-miR-6615-3p & ENSGALT00000043224 \\
TCONS_00040913 & gga-miR-6615-3p & ENSGALT00000050730 \\
TCONS_00038747 & gga-miR-6615-3p & ENSGALT00000050730 \\
TCONS_00038748 & gga-miR-6615-3p & ENSGALT00000050730 \\
TCONS_00055280 & gga-miR-135a-5p & JAM3 \\
TCONS_00055280 & gga-miR-135a-5p & GPR133 \\
TCONS_00055280 & gga-miR-135a-5p & CLDN1 \\
TCONS_00055280 & gga-miR-135a-5p & TMEM123 \\
\hline
\end{tabular}



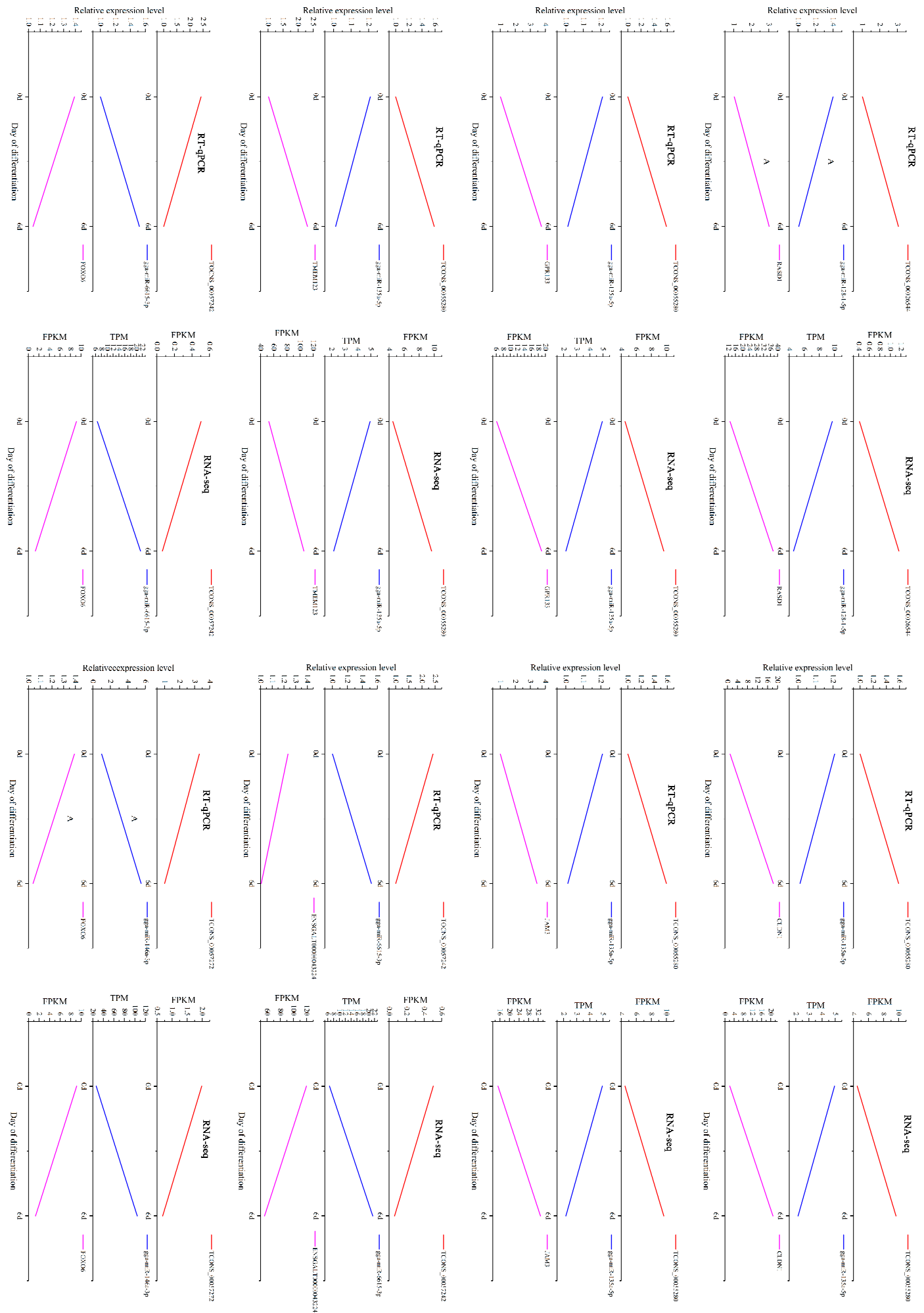

Figure 7. Validation of crucial ceRNA interactions using quantitative reverse transcription polymerase chain reaction (RT-qPCR). Red, blue, and pink lines indicate lncRNA, miRNA, and mRNA, respectively. Eight out of twelve ceRNA interactions were validated using RT-qPCR, including TCONS_00026544-gga-miR-128-1-5p-RASD1, TCONS_00057272-gga-miR-146a-3p-FOXO6, TCONS_00057242-gga-miR-6615-3p-FOXO6, TCONS_00057242-gga-miR-6615-3p-ENSGALT000000 43224, TCONS_00055280-gga-miR-135a-5p-JAM3， TCONS_00055280-gga-miR-135a-5p-GPR133, TCONS_00055280-gga-miR-135a-5p-CLDN1, TCONS_00055280-gga-miR-135a-5p-TMEM123. 


\section{Discussion}

In mammals, the molecular and cellular mechanisms underlying adipose tissue development have been well studied. The development of adipose tissue is a result of both increased number of new adipocytes (hyperplasia) and increased deposition of lipid in adipocytes (hypertrophy) [32]. However, adipose tissue development in chickens is still poorly understood. lncRNA was originally thought to be noise of RNA polymerase II transcription because it cannot encode a protein [33]. In recent years, with the development of high-throughput sequencing technology, more and more lncRNAs have been annotated. Increasing evidence has confirmed that lncRNAs are widely involved in the regulation of gene expression [34], cell proliferation [35], cell differentiation [36], cell apoptosis [37], and cancer development [38].

Recent studies suggest a significant number of lncRNAs play vital roles in regulating adipogenesis. For instance, IncRNA Lnc-U90926 attenuates 3T3-L1 adipocyte differentiation via inhibiting the transactivation of PPAR $\gamma 2$ or PPAR $\gamma$ [39]. PU.1 AS IncRNA, a novel AS IncRNA transcripted from the porcine PU.1 gene promotes adipogenesis through the formation of a sense-antisense RNA duplex with PU.1 mRNA [7]. However, the roles of lncRNAs in chicken adipogenesis remain elusive. To investigate the function of lncRNAs in chicken adipogenesis, we firstly isolated and cultured the preadipocytes from chicken abdominal fat tissue. Chicken preadipocytes were induced to differentiation in vitro using differentiation medium supplemented with oleic acid. Oil red O staining and RT-qPCR of adipocyte differentiation marker genes indicated that the chicken preadipocyte in vitro induction model was successfully constructed. We characterised the expression profiles of lncRNAs during the differentiation of chicken preadipocytes using Ribo-zero RNA-seq based on the in vitro induction model. A total of 3874 lncRNAs were identified, in which 235 lncRNAs were differentially expressed in the differentiation of chicken preadipocytes. Of the 235 DELs, 169 lncRNAs were up-regulated after differentiation, suggesting that most lncRNAs might play positive roles in the regulation of chicken preadipocytes differentiation. We also identified 145 DEMis and 660 DEMs between undifferentiated and differentiated preadipocytes. We found that top DEMs, such as FABP4 [40] and KLF5 [41] are involved in adipose tissue development of chicken. In the DEMis, gga-miR-128 [42] and gga-miR-223 [43] participate in regulating chicken preadipocytes differentiation. These differentially expressed genes were considered as potential key regulators in the differentiation of chicken preadipocytes.

Growing evidence suggests that lncRNAs can act as ceRNAs to indirectly regulate mRNAs by competitively binding miRNAs $[10,15,44]$. lncRNA-associated ceRNA networks in human adipocyte differentiation have been constructed $[45,46]$, and lncRNAs regulating adipocyte differentiation as ceRNA in human [13], bovine [15], and mouse [47] have been identified. Nevertheless, the specific lncRNA-associated ceRNA network involved in the differentiation of chicken preadipocytes is yet largely unknown. In this study, we mainly focused on the construction of lncRNA-associated ceRNA network related to chicken preadipocytes differentiation. We firstly identified 235 DELs, 145 DEMis, and 660 DEMs between differentiated and undifferentiated preadipocytes. By target prediction and expression correlation analysis, 251 ceRNAs (lncRNA-miRNA-mRNA) $(p<0.05)$ of 96 DELs, 71 DEMis, and 84 DEMs were identified. We then constructed a ceRNA network based on these 251 ceRNA triples. To characterize the underlying function of the ceRNA network, we performed GO and KEGG pathway analysis on the 84 DEMs. GO analysis results revealed that DEMs were significantly enriched in positive regulation of cell differentiation biological process, suggesting the potential role of the constructed ceRNA network in cell differentiation. Pathway analysis also showed that DEMs were enriched in pathways related to preadipocyte differentiation, including pantothenate and CoA biosynthesis and steroid hormone biosynthesis.

To date, the function of lncRNA in chicken is poorly annotated. It is challenging to identify key lncRNA directly by its function. In our study, we identify crucial lncRNA-miRNA-mRNA interactions by miRNAs involved in adipogenesis and miRNAs with high connection degree and expression. Studies showed that miR-128 promoted adipogenic differentiation of human mesenchymal stem cells by suppression of VEGF pathway [29]. The miR-146a can promote BM-MSC to differentiate into 
adipocytes [30]. miR-135a-5p inhibits 3T3-L1 adipogenesis through activation of canonical Wnt $/ \beta$-catenin signaling [31]. gga-miR-6615-3p were high-connected in the network and high-expressed in chicken preadipocytes. These four miRNAs may play crucial roles in the differentiation of chicken preadipocytes. Finally, we identified 12 vital lncRNA-miRNA-mRNA interactions containing the above four miRNAs from the lncRNA-associated ceRNA network. Eight out of the twelve interactions were validated by RT-qPCR: TCONS_00026544-gga-miR-128-1-5p-RASD1, TCONS_00057272-gga-miR-146a-3p-FOXO6, TCONS_00057242-gga-miR-6615-3p-FOXO6, TCONS_00057242-gga-miR-6615-3p-ENSGALT00000043224, TCONS_00055280-gga-miR-135a-5p-JAM3， TCONS_00055280-gga-miR-135a-5p-GPR133， TCONS _00055280-gga-miR-135a-5p-CLDN1, TCONS_00055280-gga-miR-135a-5p-TMEM123. The expression of gga-miR-128-1-5p and gga-miR-135a-5p decreased during the differentiation of chicken preadipocytes. It suggests that lncRNA TCONS_00026544 and TCONS_00055280 may promote adipogenic differentiation of chicken preadipocytes by sponging gga-miR-128-1-5p and gga-miR-135a-5p, thereby up-regulating the expression of target genes, RASD1, JAM3, GPR133, CLDN1, and TMEM123. Instead, gga-miR-146a-3p and gga-miR-6615-3p were up-regulated in differentiated cells, demonstrating their positive role in regulating the adipogenesis of chicken. IncRNA TCONS_00057272 and TCONS_00057242 may sponge gga-miR-146a-3p and gga-miR-6615-3p as ceRNA, increase the expression of their target genes FOXO6 and ENSGALT00000043224, thereby inhibit the differentiation of chicken preadipocytes. However, the contribution of the crucial lncRNA-miRNA-mRNA interaction identified in our study is still not certain. Further studies should be performed to address these issues.

\section{Conclusions}

In summary, we systematically analyzed the differentially expressed lncRNAs, miRNAs, and mRNAs between differentiated and undifferentiated preadipocytes using RNA-seq and miRNA-seq. We then constructed the lncRNA-associated ceRNA network following the ceRNA hypothesis. Eight crucial ceRNA interactions were identified based on the interacted miRNAs function and RT-qPCR. TCONS_00026544-gga-miR-128-1-5p-RASD1, TCONS_00055280-gga-miR-135a-5p-JAM3, TCONS_00055280-gga-miR-135a-5p-GPR133, TCONS_00055280-gga-miR-135a-5p-CLDN1, and TCONS _00055280-gga-miR-135a-5p-TMEM123 may promote adipogenic differentiation of chicken preadipocytes while TCONS_00057272-gga-miR-146a-3p-FOXO6, TCONS_00057242-gga-miR-6615-3p-FOXO6, and TCONS_00057242-gga-miR-6615-3p-ENSGALT00000043224 have the opposite effects. Our study constructs a ceRNA regulatory network and identifies eight crucial ceRNA interactions related to chicken preadipocytes differentiation, which will lead to a better understanding of chicken fat deposition. However, the ceRNA network and crucial ceRNA interactions is results of prediction based on gene expression and target prediction, and their function in the differentiation of chicken preadipocytes still needs to be verified by experiments.

Supplementary Materials: The following are available online at http://www.mdpi.com/2073-4425/10/10/795/s1, Data S1: The arguments and the thresholds used to identify lncRNAs and miRNAs, Data S2: Normal distribution test of RT-qPCR data of PPARG and FABP4, Data S3: Sequences of lncRNAs in chicken preadipocytes, Table S1: Differentially expressed lncRNAs, miRNAs, and mRNAs, Table S2: Expression correlation analysis of DELs, DEMis, and DEMs, Table S3: Potential ceRNA interactions in the differentiation of chicken preadipocytes.

Author Contributions: Conceptualization, T.Z.; Data curation, L.C., T.Z., J.H., G.Z., and G.D.; Formal analysis, L.C., T.Z., and G.Z.; Funding acquisition, K.X., J.W., and H.W.; Investigation, L.C., T.Z., S.Z. and J.H.; Methodology, L.C.; Resources, K.X. and J.W.; Software, T.Z.; Validation, S.Z.; Visualization, T.Z.; Writing-original draft, L.C. and T.Z.; Writing—review and editing, T.Z. and G.D.

Funding: This research was funded by the Priority Academic Program Development of Jiangsu Higher Education Institutions, the China Agriculture Research System (CARS-41), the Natural Science Foundation of Jiangsu Province (BK20180909), the Project of Natural Science Research in College and University of Jiangsu Province (18KJB230006), and the Natural Science Foundation of Yangzhou City (YZ2018096).

Conflicts of Interest: The authors declare no conflict of interest. 


\section{References}

1. Knowles, T.G.; Kestin, S.C.; Haslam, S.M.; Brown, S.N.; Green, L.E.; Butterworth, A.; Pope, S.J.; Pfeiffer, D.; Nicol, C.J. Leg disorders in broiler chickens: Prevalence, risk factors and prevention. PLoS ONE 2008, 3, e1545. [CrossRef] [PubMed]

2. Zhou, H.; Deeb, N.; Evock-Clover, C.M.; Ashwell, C.M.; Lamont, S.J. Genome-wide linkage analysis to identify chromosomal regions affecting phenotypic traits in the chicken. II. Body composition. Poult. Sci. 2006, 85, 1712-1721. [CrossRef] [PubMed]

3. Sahraei, M. Feed restriction in broiler chickens production. Biotechnol. Anim. Husb. 2012, 28, 333-352. [CrossRef]

4. Braun, E.J.; Sweazea, K.L. Glucose regulation in birds. Comp. Biochem. Physiol. B Biochem. Mol. Biol. 2008, 151, 1-9. [CrossRef]

5. Vienberg, S.; Geiger, J.; Madsen, S.; Dalgaard, L.T. MicroRNAs in metabolism. Acta Physiol. 2017, $219,346-361$. [CrossRef]

6. Bhaskaran, M.; Mohan, M. MicroRNAs: History, biogenesis, and their evolving role in animal development and disease. Vet. Pathol. 2014, 51, 759-774. [CrossRef]

7. Wei, S.; Du, M.; Jiang, Z.; Hausman, G.J.; Zhang, L.; Dodson, M.V. Long noncoding RNAs in regulating adipogenesis: New RNAs shed lights on obesity. Cell. Mol. Life Sci. 2016, 73, 2079-2087. [CrossRef]

8. Tang, H.; Chen, Y.; Zhou, H. [Research progress of long noncoding RNA in regulating adipogenesis]. Zhong Nan Da Xue Xue Bao Yi Xue Ban 2018, 43, 912-919. [CrossRef]

9. Salmena, L.; Poliseno, L.; Tay, Y.; Kats, L.; Pandolfi, P.P. A ceRNA hypothesis: The Rosetta Stone of a hidden RNA language? Cell 2011, 146, 353-358. [CrossRef]

10. Liu, H.; Zhang, Z.; Wu, N.; Guo, H. Integrative Analysis of Dysregulated lncRNA-Associated ceRNA Network Reveals Functional lncRNAs in Gastric Cancer. Genes 2018, 9, 303. [CrossRef]

11. Pilyugin, M.; Irminger-Finger, I. Long non-coding RNA and microRNAs might act in regulating the expression of BARD1 mRNAs. Int. J. Biochem. Cell B. 2014, 54, 356-367. [CrossRef] [PubMed]

12. Liu, W.; Ma, C.; Yang, B.; Yin, C.; Zhang, B.; Xiao, Y. LncRNA Gm15290 sponges miR-27b to promote PPAR $\gamma$-induced fat deposition and contribute to body weight gain in mice. Biochem. Biophys. Res. Commun. 2017, 493, 1168-1175. [CrossRef] [PubMed]

13. Li, M.; Xie, Z.; Wang, P.; Li, J.; Liu, W.; Tang, S.; Liu, Z.; Wu, X.; Wu, Y.; Shen, H. The long noncoding RNA GAS5 negatively regulates the adipogenic differentiation of MSCs by modulating the miR-18a/CTGF axis as a ceRNA. Cell Death Dis. 2018, 9, 554. [CrossRef] [PubMed]

14. Liu, H.; Li, H.; Jin, L.; Li, G.; Hu, S.; Ning, C.; Guo, J.; Shuai, S.; Li, X.; Li, M. Long Noncoding RNA GAS5 Suppresses 3T3-L1 Cells Adipogenesis Through miR-21a-5p/PTEN Signal Pathway. DNA Cell Biol. 2018, 37, 767-777. [CrossRef] [PubMed]

15. Li, M.; Sun, X.; Cai, H.; Sun, Y.; Plath, M.; Li, C.; Lan, X.; Lei, C.; Lin, F.; Bai, Y.; et al. Long non-coding RNA ADNCR suppresses adipogenic differentiation by targeting miR-204. Biochim. Biophys. Acta 2016, 1859, 871-882. [CrossRef] [PubMed]

16. Shang, Z.; Guo, L.; Wang, N.; Shi, H.; Wang, Y.; Li, H. Oleate promotes differentiation of chicken primary preadipocytes in vitro. Biosci. Rep. 2014, 34, e00093. [CrossRef] [PubMed]

17. Langmead, B.; Salzberg, S.L. Fast gapped-read alignment with Bowtie 2. Nat. Methods 2012, 9, 357-359. [CrossRef]

18. Kim, D.; Pertea, G.; Trapnell, C.; Pimentel, H.; Kelley, R.; Salzberg, S.L. TopHat2: Accurate alignment of transcriptomes in the presence of insertions, deletions and gene fusions. Genome Biol. 2013, 14, R36. [CrossRef]

19. Kong, L.; Zhang, Y.; Ye, Z.Q.; Liu, X.Q.; Zhao, S.Q.; Wei, L.; Gao, G. CPC: Assess the protein-coding potential of transcripts using sequence features and support vector machine. Nucleic Acids Res. 2007, 35, W345-W349. [CrossRef]

20. Sun, L.; Luo, H.; Bu, D.; Zhao, G.; Yu, K.; Zhang, C.; Liu, Y.; Chen, R.; Zhao, Y. Utilizing sequence intrinsic composition to classify protein-coding and long non-coding transcripts. Nucleic Acids Res. 2013, 41, e166. [CrossRef]

21. Bairoch, A.; Apweiler, R. The SWISS-PROT protein sequence database and its supplement TrEMBL in 2000. Nucleic Acids Res. 2000, 28, 45-48. [CrossRef] [PubMed] 
22. Benson, D.A.; Karsch-Mizrachi, I.; Clark, K.; Lipman, D.J.; Ostell, J.; Sayers, E.W. GenBank. Nucleic Acids Res. 2012, 40, D48-D53. [CrossRef] [PubMed]

23. Kalvari, I.; Argasinska, J.; Quinones-Olvera, N.; Nawrocki, E.P.; Rivas, E.; Eddy, S.R.; Bateman, A.; Finn, R.D.; Petrov, A.I. Rfam 13.0: Shifting to a genome-centric resource for non-coding RNA families. Nucleic Acids Res. 2018, 46, D335-D342. [CrossRef] [PubMed]

24. Robinson, M.D.; McCarthy, D.J.; Smyth, G.K. edgeR: A Bioconductor package for differential expression analysis of digital gene expression data. Bioinformatics 2010, 26, 139-140. [CrossRef] [PubMed]

25. Wong, N.; Wang, X. miRDB: An online resource for microRNA target prediction and functional annotations. Nucleic Acids Res. 2015, 43, D146-D152. [CrossRef]

26. Agarwal, V.; Bell, G.W.; Nam, J.W.; Bartel, D.P. Predicting effective microRNA target sites in mammalian mRNAs. eLife 2015, 4, e05005. [CrossRef]

27. Xu, X.W.; Zhou, X.H.; Wang, R.R.; Peng, W.L.; An, Y.; Chen, L.L. Functional analysis of long intergenic non-coding RNAs in phosphate-starved rice using competing endogenous RNA network. Sci. Rep. 2016, 6, 20715. [CrossRef]

28. Yu, G.; Wang, L.G.; Han, Y.; He, Q.Y. clusterProfiler: An R package for comparing biological themes among gene clusters. Omics 2012, 16, 284-287. [CrossRef]

29. Chen, C.; Deng, Y.; Hu, X.; Ren, H.; Zhu, J.; Fu, S.; Xie, J.; Peng, Y. miR-128-3p regulates 3T3-L1 adipogenesis and lipolysis by targeting Pparg and Sertad2. J. Physiol. Biochem. 2018, 74, 381-393. [CrossRef]

30. Ye, X.; Wang, X.B.; Wang, J.; Ming, J. Effect of MicroRNA-146a on Differentiation Potential of Human Bone Marrow Mesenchymal Stem Cells. Zhongguo Shi Yan Xue Ye Xue Za Zhi 2016, 24, 596-601. [CrossRef]

31. Chen, C.; Peng, Y.; Peng, Y.; Peng, J.; Jiang, S. miR-135a-5p inhibits 3T3-L1 adipogenesis through activation of canonical Wnt/ $\beta$-catenin signaling. J. Mol. Endocrinol. 2014, 52, 311-320. [CrossRef] [PubMed]

32. Wang, G.; Kim, W.K.; Cline, M.A.; Gilbert, E.R. Factors affecting adipose tissue development in chickens: A review. Poul. Sci. 2017, 96, 3687-3699. [CrossRef] [PubMed]

33. Ponting, C.P.; Oliver, P.L.; Reik, W. Evolution and functions of long noncoding RNAs. Cell 2009, 136, 629-641. [CrossRef] [PubMed]

34. Sun, Q.; Hao, Q.; Prasanth, K.V. Nuclear Long Noncoding RNAs: Key Regulators of Gene Expression. Trends Genet. 2018, 34, 142-157. [CrossRef] [PubMed]

35. Sun, P.; Wang, J.; Guo, X.; Chen, Y.; Xing, C.; Gao, A. Benzene and its metabolite decreases cell proliferation via LncRNA-OBFC2A-mediated anti-proliferation effect involving NOTCH1 and KLF15. Oncotarget 2017, 8, 40857-40871. [CrossRef]

36. Fatica, A.; Bozzoni, I. Long non-coding RNAs: New players in cell differentiation and development. Nat. Rev. Genet. 2014, 15, 7-21. [CrossRef]

37. Chen, L.; Yang, W.; Guo, Y.; Chen, W.; Zheng, P.; Zeng, J.; Tong, W. Exosomal lncRNA GAS5 regulates the apoptosis of macrophages and vascular endothelial cells in atherosclerosis. PLoS ONE 2017, 12, e0185406. [CrossRef]

38. Bhan, A.; Soleimani, M.; Mandal, S.S. Long Noncoding RNA and Cancer: A New Paradigm. Cancer Res. 2017, 77, 3965-3981. [CrossRef]

39. Chen, J.; Liu, Y.; Lu, S.; Yin, L.; Zong, C.; Cui, S.; Qin, D.; Yang, Y.; Guan, Q.; Li, X.; et al. The role and possible mechanism of lncRNA U90926 in modulating 3T3-L1 preadipocyte differentiation. Int. J. Obes. 2017, 41, 299-308. [CrossRef]

40. Shipp, S.L.; Cline, M.A.; Gilbert, E.R. Promotion of adipogenesis by neuropeptide Y during the later stages of chicken preadipocyte differentiation. Physiol. Rep. 2016, 4, e13006. [CrossRef]

41. Wang, W.; Cheng, M.; Qiao, S.; Wang, Y.; Li, H.; Wang, N. Gga-miR-21 inhibits chicken pre-adipocyte proliferation in part by down-regulating Kruppel-like factor 5. Poul. Sci. 2017, 96, 200-210. [CrossRef] [PubMed]

42. Zhang, M.; Li, F.; Sun, J.W.; Li, D.H.; Li, W.T.; Jiang, R.R.; Li, Z.J.; Liu, X.J.; Han, R.L.; Li, G.X.; et al. LncRNA IMFNCR Promotes Intramuscular Adipocyte Differentiation by Sponging miR-128-3p and miR-27b-3p. Front. Genet. 2019, 10, 42. [CrossRef] [PubMed]

43. Li, F.; Li, D.; Zhang, M.; Sun, J.; Li, W.; Jiang, R.; Han, R.; Wang, Y.; Tian, Y.; Kang, X.; et al. miRNA-223 targets the GPAM gene and regulates the differentiation of intramuscular adipocytes. Gene 2019, 685, 106-113. [CrossRef] [PubMed] 
44. Ji, D.; Hu, G.; Zhang, X.; Yu, T.; Yang, J. Long non-coding RNA DSCAM-AS1 accelerates the progression of hepatocellular carcinoma via sponging miR-338-3p. Am. J. Transl. Res. 2019, 11, 4290-4302. [PubMed]

45. Chen, K.; Xie, S.; Jin, W. Crucial lncRNAs associated with adipocyte differentiation from human adipose-derived stem cells based on co-expression and ceRNA network analyses. PeerJ 2019, 7, e7544. [CrossRef] [PubMed]

46. Guo, Z.; Cao, Y. An lncRNAmiRNAmRNA ceRNA network for adipocyte differentiation from human adiposederived stem cells. Mol. Med. Rep. 2019, 19, 4271-4287. [CrossRef]

47. Wang, Y.; Liu, W.; Liu, Y.; Cui, J.; Zhao, Z.; Cao, H.; Fu, Z.; Liu, B. Long noncoding RNA H19 mediates LCoR to impact the osteogenic and adipogenic differentiation of mBMSCs in mice through sponging miR-188. J. Cell. Physiol. 2018, 233, 7435-7446. [CrossRef]

(C) 2019 by the authors. Licensee MDPI, Basel, Switzerland. This article is an open access article distributed under the terms and conditions of the Creative Commons Attribution (CC BY) license (http://creativecommons.org/licenses/by/4.0/). 